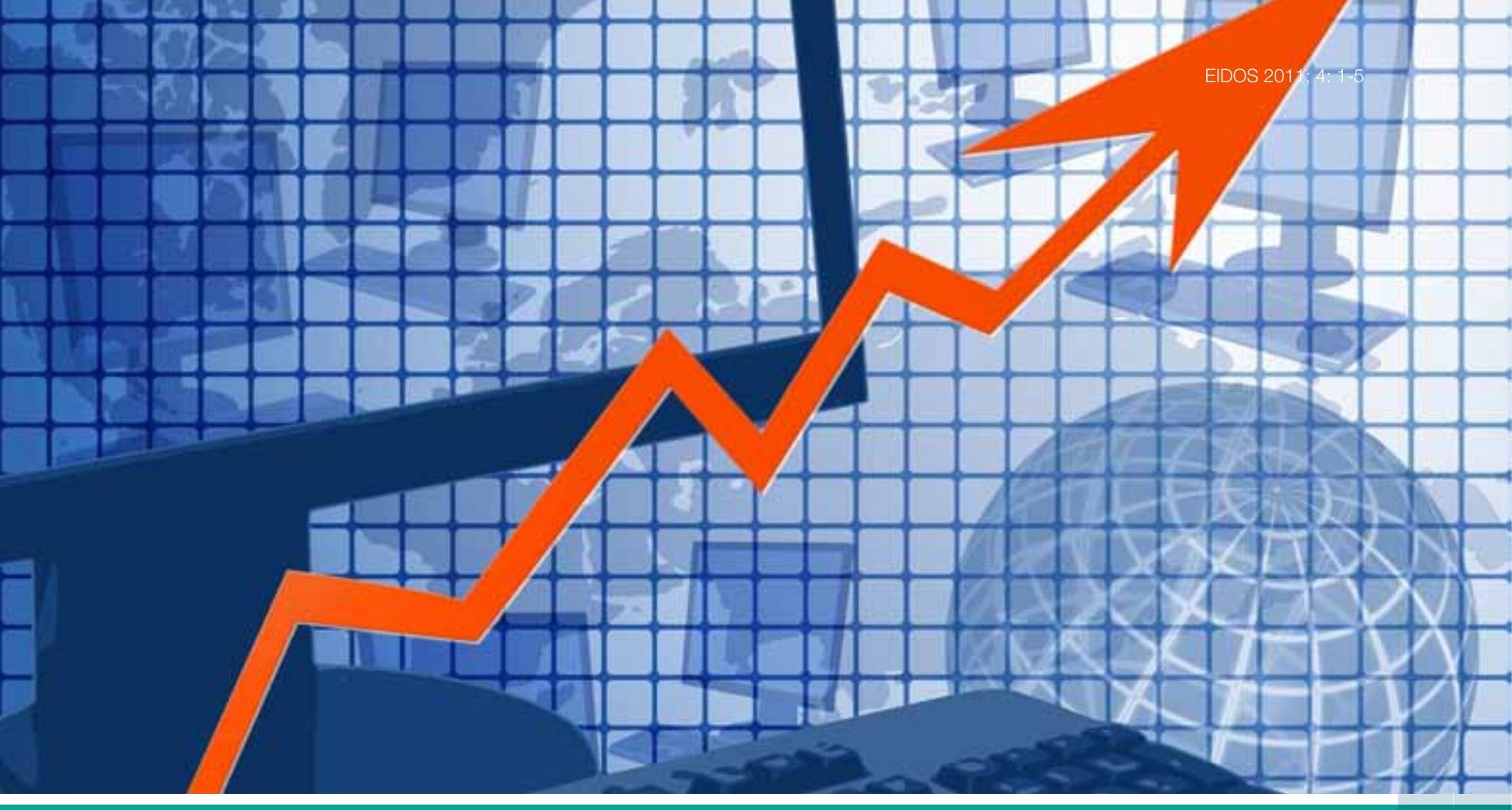

EIDOS, 4

Marzo-agosto 2011, 69-73

ISSN:1390-499X

elSSN:1390-5007

\title{
SISTEMA SOPOG, PARA ELABORACIÓN PRIMARIA DE DATOS ESTADÍSTICOS
}

O. Chadrina, F. Freire ${ }^{1}$, Universidad Tecnológica Equinoccial

Recepción/Received: 2011-01-07

Aceptación/Accepted: 2011-07-12

Publicado/Published: 2011-08-05

Resumen -Este artículo describe el desarrollo de un sistema informático automatizado, para la definición de los parámetros del conjunto general homogéneo (SOPOG), destinado a la elaboración primaria de la información, previo su utilización en Sistemas GIS, que permite incrementar considerablemente la calidad de los datos estadísticos.

Palabras clave -Conjunto General Homogéneo, Java, UML

Abstract -This article describes the development of a computerized informatics system, for defining the benchmarks of homogeneous general group (SOPOG), allocated to primary elaboration of information, considering its previous use on GIS systems that makes possible to increase considerably statistics data quality.

Keywords -General Homogeneous set, Java, UML

1 Fausto Freire Carrera; ffreire@ute.edu.ec; Docente de Posgrado Universidad Tecnológica Equinoccial 


\section{INTRODUCCIÓN}

Las tecnologías de telecomunicación en la actualidad tienen diversas aplicaciones, incluso se utilizan en el monitoreo y pronostico de desastres, sea estos provocados por el hombre o de carácter natural. Particularmente han encontrado aplicación en sistemas de Geo-informativos (GIS), que permiten recopilar información, sobre el medio ambiente circundante en la formato digital, representada en forma de mapas electrónicos dinámicos, permitiendo elaborar rápidamente la información y difundirla en régimen de tiempo real. Los sistemas GIS realizan el tratamiento completo de la información como: recopilación, almacenamiento, actualización y representación. Por consiguiente, en la actualidad la elaboración de métodos primarios del tratamiento de la información que incluyan la valoración de las probabilidades, la independencia, la homogeneidad y representación de las muestras en las áreas investigadas, que hasta la actualidad se han basado en hipótesis a priori. En los trabajos [1-3] por primera vez se propone resolver este problema.

En base a los algoritmos desarrollados en los trabajos $[2,3]$ fue desarrollado el sistema informático $\left(\right.$ SOPOG $\left.^{2}\right)$, para la elaboración primaria de la información estadística con muestras limitadas que permite estimar la homogeneidad de la muestra y su representatividad, como también permite determinar los parámetros de los conjuntos generales homogéneos y escoger los que más se ajusten a los criterios de selección de todos los posibles.

En la última década se ha intensificado la tendencia de la simulación de sistemas complejos mediante la creación de modelos gráficos, especialmente para desarrollo de software.

En este trabajo se utilizo el lenguaje de unificado de modelado UML [4], para el diseño y la documentación del sistema informático, mediante la definición de los diagramas de estructura, comportamiento e interacción.

En la figura 1 se muestra el diagrama de "casos de uso" donde el usuario llamado en el diagrama de "investigador", ejecuta el programa principal (el nodo "Inicio"). Antes de ingresar los datos es necesarios seleccionar entre el cálculo con un número fijo de muestras (múltiplos de cuatro, el

2 Patente № 2006613375 KSTU (RU)

c) nodo "Entrada de datos (») y un número variable de muestras (no múltiplos de cuatro, nodo "Entrada de datos II»).

Luego el cálculo se realiza en dos subrutinas diferentes: los nodos "Procesamiento de Datos |» y Procesamiento de Datos II ». Al finalizar los cálculos, el sistema proporciona automáticamente los resultados en forma grafica y de tablas, para la toma de decisiones.

Figura 1. El diagrama como «use case» el sistema SOPOG informativo

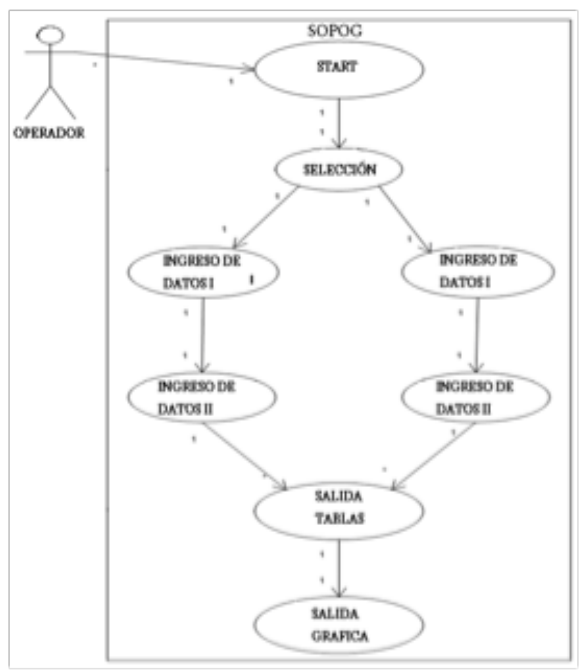

En la figura 2 se presentan los diagramas de «clase», rutinas de arranque, construcción de gráficas e histogramas de sistema "SOPOG. En estos diagramas muestran las clases, interfaces, objetos, sus relaciones.

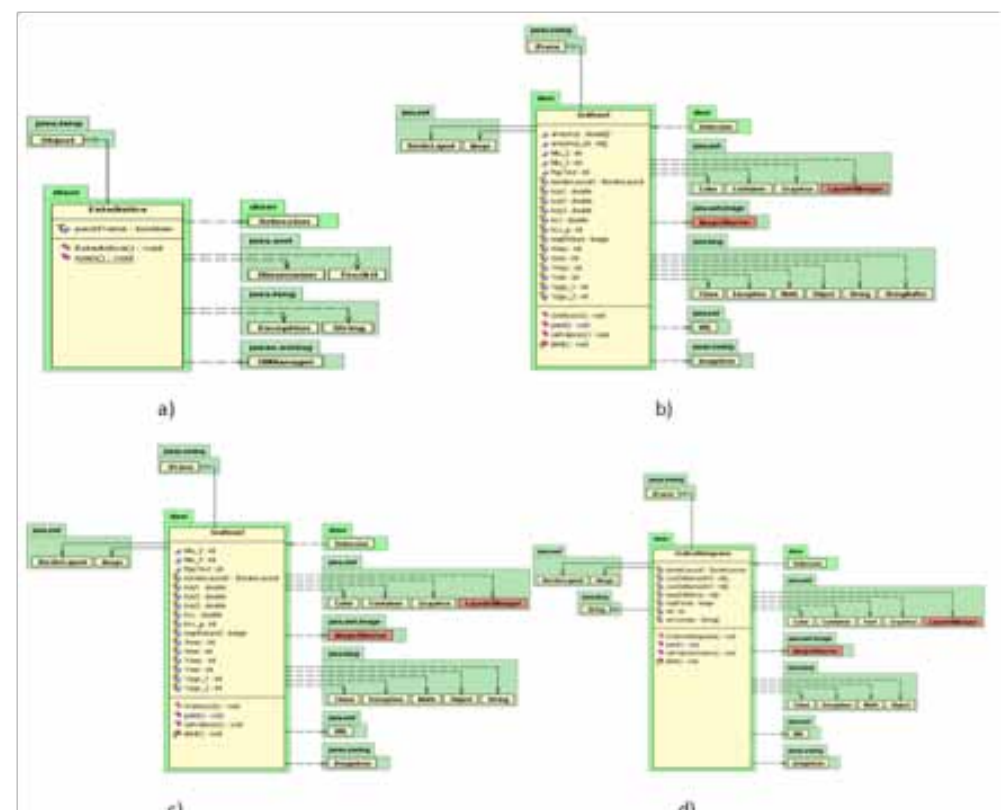

Figura 2. Diagramas de clases; a) subprograma de inicio del sistema, b) y c) diagramas de clases para construcción de los gráficos; c) diagrama de clase para construcción de histogramas 
Las clases de construcción de los gráficos e histogramas del sistema (Graficos1, Graficos2, GraficoHistograma) abastecen la salida de los datos en la forma gráfica.

El sistema SOPOG fue desarrollado en Java. Lenguaje orientado a objetos, que permite desarrollar programas eficaces y seguros para trabajar en ambientes web.

Al igual que los lenguajes programación exitosos anteriores; Java heredo los mejores elementos e innovaciones implementadas que le dan su fortaleza [5].

El lenguaje de programación Java tiene ventajas significativas ante otros lenguajes, e incluso ante sus antecesores, en la simplicidad, seguridad, movilidad, es de orientación a objetos, estabilidad, programación en hilos, independencia arquitectónica, integración y una alta eficiencia.

Para el desarrollo de la interface de usuario del sistema se utilizó JBuilderX. Los potentes constructores visuales permiten agilizar el proceso de elaboración de los componentes y especialmente acelerar el proceso del desarrollo en implementación en los servidores de aplicaciones. El ambiente de programación JBuilderX permite trabajar con bases de datos, posee un sistema de control de versiones de las fuentes, modelación visual, pruebas y generación de documentación del código.

Una vez iniciado el sistema se deben ingresar los valores estadísticos recogidos en las investigaciones (el número de datos no debe ser menor a cuatro), utilizando la venta de la figura 3.

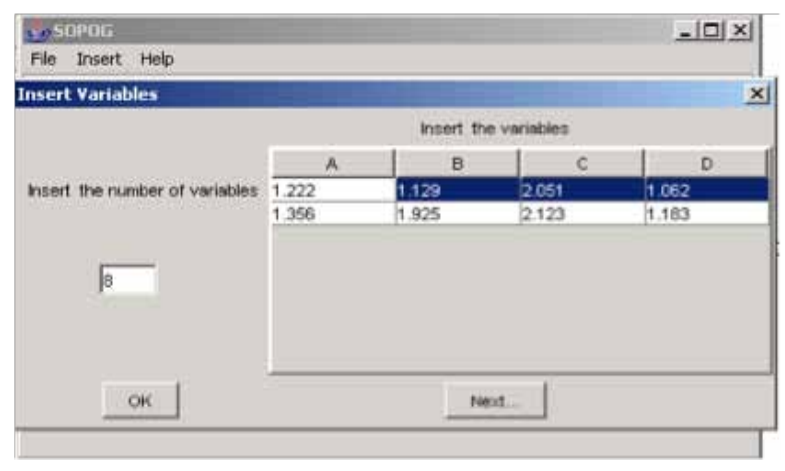

Figura 3. Ventana de ingreso de datos múltiples de cuatro

Cuando la cantidad de los datos no es múltiple de cuatro, la introducción se realiza mediante la interface que se presenta en la siguiente figura.

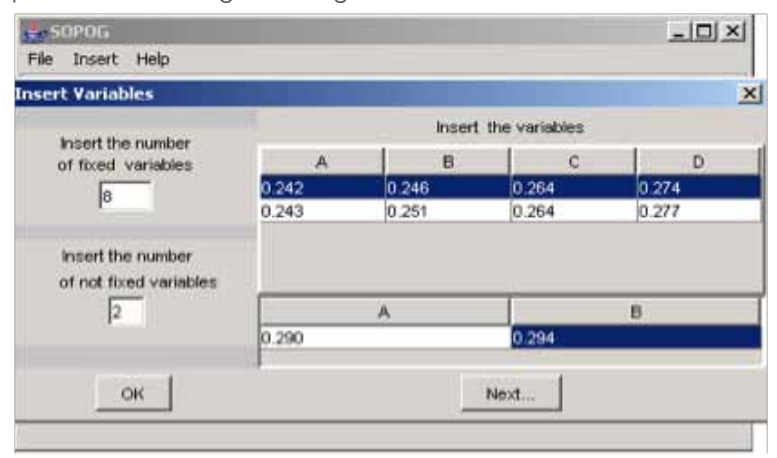

Figura 4. La interface de introducción de datos no múltiples de cuatro

El sistema en el régimen automatizado realiza la evaluación de la muestra sobre la homogeneidad y representatividad. En caso de que la muestra corresponda, se procede con el cálculo del parámetro Rgs.

En las figura 6 se refleja los resultados de los cálculos realizados, cuando la muestra es múltiple cuatro. En el ejemplo examinado la muestra Yi es homogénea y representativa con un parámetro Rgs=0,39.

Una de las características del sistema es la posibilidad del tratamiento de cualquier muestra, no solamente múltiple cuatro que se propuesto en los trabajos [2,3].

En la figura 5 se presenta el algoritmo generalizado para la formación de las combinaciones de la nuestras, cuyo número de elementos no es múltiple a cuatro.

El sistema calcula la cantidad de combinaciones obtenidas en caso que haya datos no fijados (pueden ser de 1 a 3) según la fórmula de Bernoulli. Luego se forman estas combinaciones y para cada uno se determina el parámetro del conjunto homogéneo general (Rgs) (distribución de Bernoulli). Luego de lo cual el Rgs obtenido se convierte en dato de entrada y como resultado el usuario obtiene el valor del Rgs definitivo.

Este tratamiento da la posibilidad de obtener un resultado más objetivo y de mejor calidad. En caso de que unas de las combinaciones no sean homogéneas y representativas, se conservan para un análisis posterior (fig. 5)

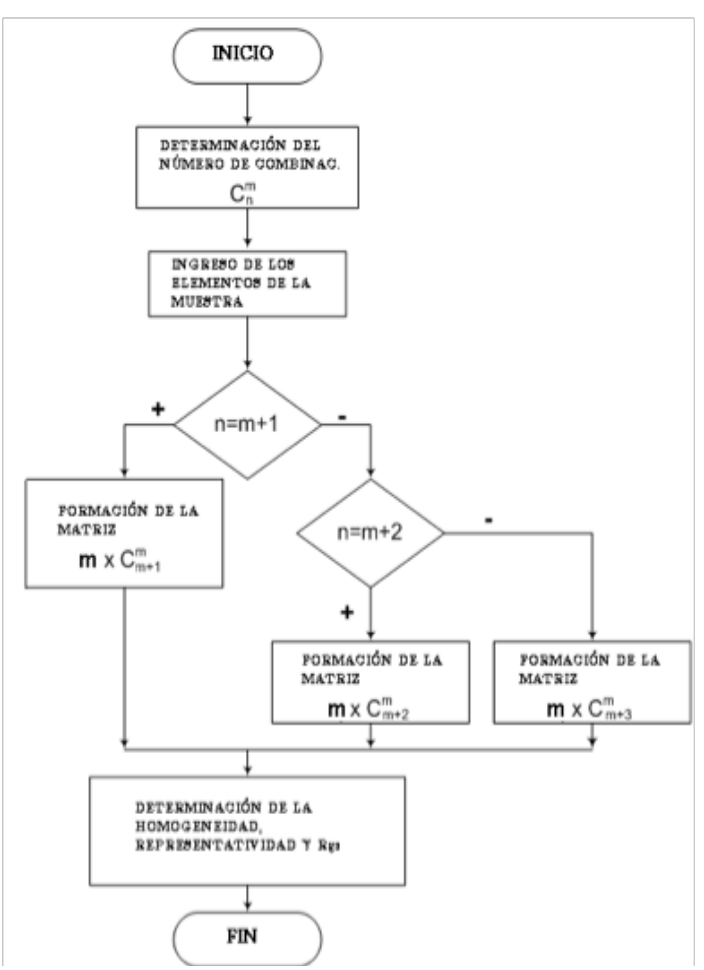

Figura 5. El algoritmo de la formación de las combinaciones de la muestra, cuando el número de elementos no es múltiple cuatro. 


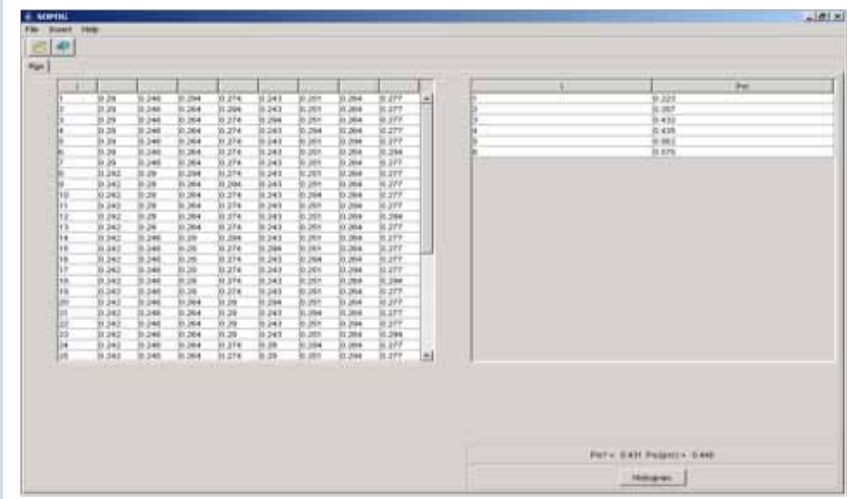

Figura 6. Resultados de los cálculos para la muestra, la cantidad de elementos no múltiples cuatro

El resultado de los cálculos utilizando datos no fijos, se presenta en el histograma Rgs de todas las combinaciones que resultaron homogéneas y representativas (fig.7)

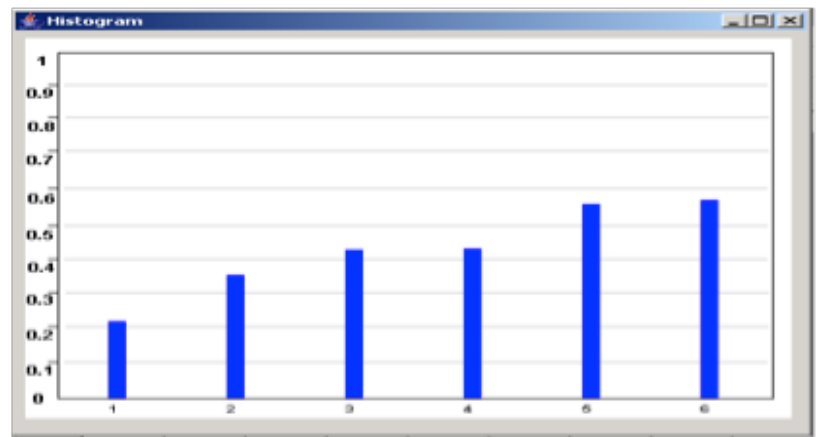

Figura 7. Histograma obtenido según los cálculos, en caso que la cantidad de los datos de la muestra no sea múltiple de cuatro.

En general, el sistema "SOPOG" está destinado para el incremento de la calidad de los datos estadísticos primarios, incluso en los sistemas que se desarrollan dinámicamente, lo cual es considerable en el uso de las tecnologías GIS.

La utilización del sistema informativo para la definición de los parámetros del conjunto general en la elaboración primaria de datos estadísticos da una visión precisa sobre representatividad y la homogeneidad de las muestras que se somete al análisis, lo cual influye positivamente en la cualidad de las investigaciones asociadas al control del riesgo ecológico y apoyo para la toma de decisiones.

\section{BIBLIOGRAFÍA}

[1] Швырков В. В. Тайна традиционной статистики запада. -М., «Финансы и статистика», 1998-139с.
[2] Уразбахтин И. Г. Уразбахтин А. И. Алгоритм проверки однородности выборки и ее репрезентативности исследуемому случайному процессу //

Инфокоммуникационные технологии. 2006. №3.

[3] Уразбахтин И. Г. Уразбахтин А. И. Алгоритм определения параметров однородных генеральных совокупностейпо репрезентативномуограниченному объему выборки из нее // Инфокоммуникационные технологии. 2006. №4.

[4] Ларман K. Применение UML и шаблонов проектирования. - М., Вильямс, 2002. - 624 с.

[5] Darwin lan. Java Cookbook, Publisher: O'Reilly, First Edition June 2001, ISBN: 0-59600-170-3, 882 pages 


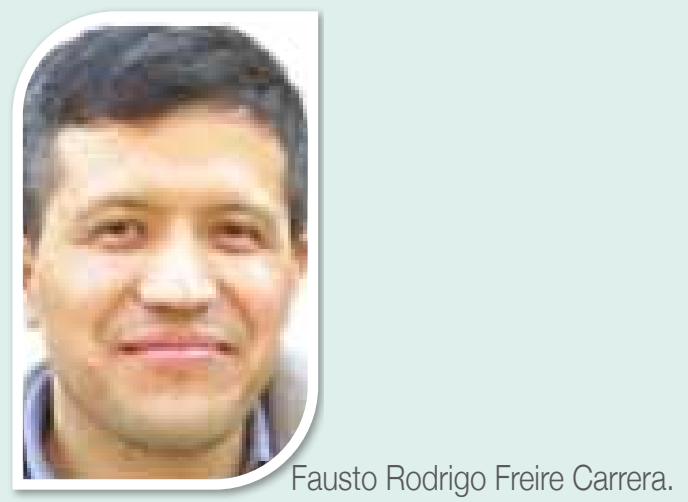

Nace en Quito en 1971; en 1995 se gradúa como Ingeniero en Sistemas Computacionales y Máster en Ciencias de Ingeniería en la Universidad Técnica Estatal de Kursk de la Federación de Rusia.

En 2001, obtiene los titulos de Máster en Tecnologías de la Información para la Fabricación y Especialista en Robótica en la Universidad Politécnica de Madrid de España, Departamento de Sistemas Inteligentes Aplicados.

En 2007, obtienen el Título: Ph.D en Dinámica, Resistencia, Aparatos y Dispositivos por la Universidad Técnica Estatal de Kursk de la Federación de Rusia, en la Facultad de Mecánica Teórica y Mecatrónica.

En la actualidad Subdirector de Posgrado de la Universidad Tecnológica Equinoccial.

Las áreas de interés son: Control Automático, Dinámica, Robótica Móvil, Inteligencia Artificial, Sistemas Distribuidos, Telemática, simulación matemática, lenguajes de Programación.

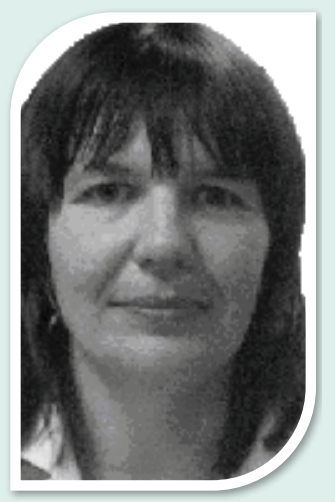

Olga Chadrina.

Nace en 1970 en la ciudad de Osh, Republica de Kirguistán. En 1995 obtiene el título de Ingeniero Químico Tecnólogo en la Especialidad Tecnología Química y Maquinaria de Producción Ingeniería en la Universidad Técnica Estatal de Kursk de la Federación de Rusia.

Desde noviembre del 2003 hasta noviembre del 2007 cursa en la Facultad de Sistemas Informáticos en Economía de la Universidad Técnica Estatal de Kursk el doctorado, obteniendo el título de Ph.D en el Manejo de Sistemas Económicos y Sociales.

Se ha desempeñado como especialista del Consern "JIMVOLOKNO", de la ciudad de Kursk, docente de la Universidad Técnica Estatal de Kursk.

Actualmente docente de la Universidad Politécnica Salesiana, en las Facultades de Ingniería Civil y Electrónica.

Las áreas de interes son: Matemáticas Superiores, la Lógica Difusa, Grafos Difusos, Probabilidades, Estadística, Cálculo Diferencial, Calculo Integral, Manejo Medioambiental y Simulación Matemática de procesos ambientales.

Cooperante en Investigación con el área de Posgra- 